\title{
Design of SMC with a Conditional Integrator for Pitch Control of a Mini UAV
}

\author{
Sajjad Hussain, Fahad Mumtaz Malik, Omair Altaf, Naseem Ahmad \\ Department of Electrical Engineering, CE\&ME, National University of Sciences and Technology \\ Islamabad, Pakistan \\ sajjad.hussain83@ee.ceme.edu.pk; malikfahadmumtaz@gmail.com; omair.altaf83@ee.ceme.edu.pk; \\ naseem.ahmad83@ee.ceme.edu.pk
}

\begin{abstract}
An SMC with a conditional integrator for pitch control with velocity regulation as a secondary objective for a mini UAV is discussed in this paper. For controller design, the longitudinal dynamics of the mini UAV are decomposed into its phugoid mode and short period mode approximations. The controller design is based on the linear model however for implementation purpose nonlinear model of longitudinal dynamics of the mini UAV is used. The robustness of the controller to disturbances because of model uncertainties and external sources are shown through extensive simulations. The results, especially the transient response shows that this controller outperforms the controller based on conventional integrators.
\end{abstract}

Keywords: Sliding Mode Control (SMC), Conditional Integrators, Unmanned Aerial Vehicle (UAV), Phugoid Mode, Short Period Mode.

\section{Introduction}

A UAV is a highly nonlinear system with complex dynamics [1]. A number of techniques have been introduced to deal with the motion of the UAVs. Traditionally the flight control system is designed by linearizing the aircraft dynamic model at numerous flight conditions and varying the control parameters or gains with respect to these flight conditions [2]. Different multivariable techniques including the structured singular value $\mu$-synthesis, $\mathrm{H}_{\infty}$ control, linear parameter varying technique (LQR/LQG) and output feedback linearization[3,4] have been used for designing control for such systems. The performance and stability of these gain scheduled approaches can be assured by the analytical frameworks developed in [5], However, the major drawback is, the performance of these controllers degrade because of external disturbances and model uncertainties. We need flight control system to be robust to model uncertainties and external disturbances. The design of robust control for the UAVs is still an open problem for research. For the improvement of robustness different nonlinear controller design have been proposed such as, dynamic inversion model described in [6], another technique that combines online adaptive neural network with model inversion control is described in [7]. Based on back stepping and neural network a nonlinear adaptive design is described in [8].In the recent past, SMC has been studied as a robust control for systems that are subjected to external disturbances and have model uncertainties [17].

Though SMC has good robustness but has some performance issues remedy of the problem is well-known replacement of the signum (sgn) function with saturation (sat) function as discussed in [12], however, saturation function induces a constant steady state error while eliminating the chattering phenomenon. To tackle this problem online parameter estimation can be deployed. Such as the design of adaptive SMC, one such technique employed for the pitch rate tracking of UAV is discussed in [9].Instead of gain scheduling that is employed in [10], this paper presents an approach for the design of a robust SMC with the capability to eliminate the steady-state error by using the integral action conditionally. The standard integral action introduces a degradation in the transient performance, to overcome that [13] has presented conditional integrators. That specific approach has been adopted in this paper for the pitch rate control design of a customized mini UAV whose design has been carried out such that optimization of the endurance is achieved pertinent details can be found in [18]. The advantage of using this method is that it provides the integral action only conditionally, which effectively eliminate chattering without degrading the response (both transient and steady state) of the system. The resulting controller is a simple high gain PI controller with anti-wind off integrator followed by saturation. 
The design of the controller is based on a simple linear model however for performance assessment, we will use the full nonlinear longitudinal model of the UAV. The longitudinal model of the UAV on which the performance of the controller is assessed is highly nonlinear MIMO system with uncertain parameters. Through extensive simulations, it is shown that this controller outperforms the stander SMC as well as the conventional integral based SMC.

The rest of the paper is organized as; section 2 explains the longitudinal model of the aircraft in section 3 the control design for the pitch rate of the aircraft is discussed, in section 4 the results obtained through simulations are discussed followed by the conclusion section.

\section{Mathematical Model}

\subsection{UAV's Equation of Motion}

In order to obtain the mathematical model for the decoupled nonlinear longitudinal equation of motion of a UAV, we will assume that the side slip angle $\beta$ and and roll angle $\varnothing$ to be zero i.e the undisturbed flight is aligned with the wind axis. Considering no thrust vectoring the 3-dof longitudinal model can be written as [14],

$$
\begin{gathered}
\dot{V}=\frac{\bar{q} S \bar{c} q}{2 m V}\left[C_{x q}(\alpha) \cos (\alpha)+C_{z q}(\alpha) \sin (\alpha)\right]-g \sin (\theta-\alpha)+\frac{\bar{q} S}{m}\left[C_{x}\left(\alpha, \delta_{e}\right) \cos (\alpha)+C_{z}\left(\alpha, \delta_{e}\right) \sin (\alpha)\right]+\frac{T}{m} \cos (\alpha), \\
\dot{\alpha}=q\left[1+\frac{\bar{q} S \bar{c}}{2 m V^{2}}\left(C_{z q}(\alpha) \cos (\alpha)-C_{x q}(\alpha) \sin (\alpha)\right)\right]+\frac{\bar{q} S}{m V}\left[C_{z}\left(\alpha, \delta_{e}\right) \cos (\alpha)-C_{x}\left(\alpha, \delta_{e}\right) \sin (\alpha)\right]+\frac{g}{V} \cos (\theta-\alpha)-\frac{T}{m V} \sin (\alpha) \\
\dot{\theta}=q, \\
\dot{q}=\frac{\bar{q} S \bar{c} q}{2 I_{y} V}\left[\bar{c} C_{m q}(\alpha)+\Delta C_{z q}(\alpha)\right]+\frac{\bar{q} S \bar{c}}{I_{y}}\left[\bar{c} C_{m}\left(\alpha, \delta_{e}\right)+\frac{\Delta}{\bar{c}} C_{z}\left(\alpha, \delta_{e}\right)\right],
\end{gathered}
$$

where $\alpha$ is the aircraft angle of attack, $q$ is the pitch rate, $T$ is the thrust generated, $\bar{q}$ is dynamic pressure, $g$ represents the earth's gravitational acceleration, $C_{m}$ is pitching moment coefficient, $C_{z}$ and $C_{x}$ is aerodynamic force coefficient along z and x-axis respectively. $C_{x q}, C_{z q}$ and $C_{m q}$ represents the change in $C_{z} C_{x}$ and $C_{m}$ w.r.t to the pitch rate ' $q$ '.

In compact form, the equation (1..4) can be written as follow,

\begin{tabular}{|c|c|c|}
\hline & $\begin{array}{l}\dot{x}=f(x)+g(x) u, \\
y=h(x),\end{array}$ & (5) \\
\hline
\end{tabular}

where $x=[V, \alpha, \theta, q]^{T}$ is the state vector, $u=[\delta e, T]^{T}$ is input and $y=[q]$ is the output. for the purpose of designing the controller we will divide the longitudinal dynamics of the model into its short period and phugoid mode however for the simulation we will always use the full nonlinear model.

The aerodynamics data for this model will be approximated using methods given in [15], for this purpose we will express all the coefficients in terms of their arguments, in body axis system they are given as,

$$
\begin{gathered}
C_{z}(\alpha, \delta e)=-C_{L}=-\left(C_{L, 0}+C_{L, \alpha} \alpha+C_{L, \delta e} \delta e\right) \\
C_{x}\left(\alpha, \delta_{e}\right)=-C_{D 0}=-\left(C_{D 0}+K C_{L}^{2}+C_{D \delta e} \delta e\right) \\
C_{m}=C_{m 0}+C_{m \alpha} \alpha+C_{m \delta \mathrm{e}} \delta \mathrm{e} \\
C_{z q}(\alpha)=\frac{\partial \mathrm{Cz}\left(\alpha, \delta_{e}\right)}{\partial q}=\frac{\partial C_{L}}{\partial q}=C_{L, q} \\
C_{x q}(\alpha)=\frac{\partial C x\left(\alpha, \delta_{e}\right)}{\partial q}=\frac{\partial C_{D}}{\partial q}=2 K C_{L} C_{L, q}+2 k C_{L, \delta e} C_{L, q} \delta_{e} .
\end{gathered}
$$

where $C_{L 0}$ is the Lift coefficient when $\alpha=0, C_{L \alpha}$ and $C_{m \alpha}$ represents the lift curve and pitching moment curve slope w.r.t $\alpha . C_{D 0}$ and $C_{m 0}$ represent the drag and pitching moment coefficient respectively when the lift generated by the aircraft is 
zero. $C_{L, q}, C_{m q}$ represents the variation of the lift coefficient and pitching moment w.r.t the pitch rate $q . C_{m \delta_{e}}$ and $C_{D \delta e}$ denotes the variation in pitching moment and drag coefficient w.r.t the elevator deflection $\delta e$.

\subsection{Linearizing the Aircraft Equation of Motion}

For linearization of the aircraft's equation of motion, we will assume that there is a small disturbance $\Delta$ in the input, states and the output such that,

$$
\Delta u=u-\hat{u}, \quad \Delta x=x-\hat{x} \text { and } \Delta y=y-\hat{y},
$$

where $\mathrm{x}, \mathrm{u}$ and $\mathrm{y}$ are as defined previously,

For any equilibrium input $\hat{u}$, there is an equilibrium state $\hat{x}$, which satisfies the following condition,

$$
f(\hat{x}, \hat{u})=0
$$

This will result in the linear approximation of the system i.e. into its short period and phugoid mode approximation as given [15],

$$
\begin{aligned}
& {\left[\begin{array}{c}
\dot{\alpha} \\
\dot{q}
\end{array}\right]=\left[\begin{array}{cc}
a_{11} & a_{12} \\
a_{21} & a_{22}
\end{array}\right]\left[\begin{array}{l}
\alpha \\
q
\end{array}\right]+\left[\begin{array}{l}
b_{1} \\
b_{2}
\end{array}\right] u} \\
& {\left[\begin{array}{c}
\dot{V} \\
\dot{\theta}
\end{array}\right]=\left[\begin{array}{ll}
a_{31} & a_{32} \\
a_{41} & a_{42}
\end{array}\right]\left[\begin{array}{l}
V \\
\theta
\end{array}\right]+\left[\begin{array}{l}
b_{3} \\
b_{4}
\end{array}\right] u}
\end{aligned}
$$

The former is known as the short period approximation and the latter as the phugoid approximation. the constants $a_{11} \ldots a_{42}$ for the short period and phugiod mode of the mini UAV were found using the methods given in [15] and are listed in table 1. The physical insight of these approximation shows that the short period mode is excited by the elevator input while the phugoid mode is excited by the thrust input to the system. Using the decoupling in expression (8 - 9) it is obvious that we can use the elevator deflection as a control input for tracking of the pitch rate command, while for regulation of velocity we will use the thrust as a control input.

Table 1: System Parameters for the short period and phugoid mode.

\begin{tabular}{|c|c|c|c|}
\hline Parameters & values & Parameters & values \\
\hline$a_{11}$ & -9.61069 & $a_{31}$ & -0.118338 \\
\hline$a_{12}$ & 2.02931 & $a_{32}$ & -0.72663 \\
\hline$a_{21}$ & -10.8428 & $a_{41}$ & 1.3586 \\
\hline$a_{22}$ & 13.589 & $a_{42}$ & 0 \\
\hline$b_{1}$ & 0.78169 & $b_{3}$ & -0.0238 \\
\hline$b_{2}$ & -30.1436 & $b_{4}$ & -4.6577 \\
\hline
\end{tabular}

\section{Control Design}

The main objective of this paper is the design of control law which will enable the mini UAV to track desired pitch rate and velocity. Let $q_{d}$ and $v_{d}$ be the desired pitch rate and velocity respectively, $e$ be the error between the desired and estimated state then,

$$
e_{1}=q-q_{d}, \quad e_{2}=v-v_{d}
$$

Form [16] we know that the sliding surface $s$ for the $\mathrm{n}^{\text {th }}$ order nonlinear system can be defined as, 


$$
s=\left(\frac{d}{d t}+k\right)^{n-1} e
$$

where $k>0$ is a constant, $n$ represent the order of the system. Here the equations of motion are represented as first-order differential equations so the surface $s$ in our case would be,

$$
s=k e .
$$

where the constant vector $k=\left[k_{1} k_{2}\right]>0$, error $e=\left(e_{1} e_{2}\right)^{T}$ and $s=\left(s_{1} s_{2}\right)^{T}$. By defining an appropriate control law which will retain the states of the system on the sliding surface it can be ensured that the error will asymptotically reach zero. In order to define a control law for the given purpose, we will use the Lyapunov direct method. A candidate Lyapunov function for this is,

$$
V=\frac{1}{2} s^{T} s
$$

Differentiating equation (13) w.r.t time will give,

$$
\dot{V}=s^{T} \dot{S},
$$

substituting values of $\dot{s}$ in equation (14) results in,

$$
\dot{V}=s^{T}\left(\begin{array}{c}
\dot{q}-\dot{q}_{d} \\
\dot{v}-\dot{v}_{d}
\end{array}\right)
$$

Now substituting values of $\dot{q}$ and $\dot{v}$ in equation (15) results in,

$$
\dot{V}=s^{T}\left(\begin{array}{l}
a_{21} \alpha+a_{22} q+b_{2} U_{2}-\dot{q}_{d} \\
a_{31} v+a_{32} \theta+b_{1} U_{1}-\dot{v}_{d}
\end{array}\right)
$$

For the system to be asymptotically stable $\dot{V}<0$, now we need to define a control law such that this condition is satisfied. For this condition to be satisfied the resulting control law consist of two parts, one which will cancel out the nominal terms known as the equivalent control law $\left(U_{1,1}, U_{2,1}\right)$ and the $2^{\text {nd }}$ part $\left(U_{1,2}, U_{2,2}\right)$ known as switching control law will take care of the uncertain terms. For our design, these laws are as given,

$$
\begin{gathered}
U_{1}=U_{1,1}+U_{1,2}=-\frac{k_{1}}{b_{1}}\left(a_{31} v+a_{32} \theta-\dot{v}_{d}\right)-\frac{k_{1}}{b_{1}}\left(a_{31} v+a_{32} \theta-\dot{v}_{d}\right) \operatorname{sgn}\left(s_{1}\right) \\
U_{2}=U_{2,1}+U_{2,2}=-\frac{k_{2}}{b_{2}}\left(a_{21} \alpha+a_{22} q-\dot{q}_{d}\right)-\frac{k_{2}}{b_{2}}\left(a_{21} \alpha+a_{22} q-\dot{q}_{d}\right) \operatorname{sgn}\left(s_{2}\right)
\end{gathered}
$$

This choice of the control law guarantees that $s$ will converge to zero and will remain there for all the future time which in turn ensures that the error $e$ will asymptotically converge to zero.

One way to simplify this controller design is that to make the coefficient of the switching term of both $U_{I}$ and $U_{2}$ constant [13] thus the resulting control laws are,

$$
\begin{gathered}
U_{1}=-\frac{k_{1}}{b_{1}}\left(a_{31} v+a_{32} \theta-\dot{v}_{d}\right)-\frac{K_{1}}{b_{1}} \operatorname{sgn}\left(s_{1}\right) \\
U_{2}==-\frac{K_{2}}{b_{2}}\left(a_{21} \alpha+a_{22} q\right)-\frac{K_{2}}{b_{2}} \operatorname{sgn}\left(s_{2}\right)
\end{gathered}
$$


Substituting this value of $U_{1}$ and $U_{2}$ in equation (16) will result in $\dot{V}<0$ which is the condition that needs to be statisfied for a system to be asymptotically stable, in addition, this condition ensures that the error converges to zero which means that the system will asymptotically track the desired pitch rate and velocity.

Any SMC designed with this control law is known as ideal SMC. The main problem with this design is chattering because of its discontinuous nature. One way to eliminate chattering is to replace the $\operatorname{sgn}($.$) (sign function) with sat(.)$ (Continuous saturation function) which leads to the steady-state error as will be shown in the simulations. To deal with the problem of steady-state error the sliding surface $s$ is augmented by an integrator which is driven by the error function $e$, the resulting sliding surface $s$ is given as,

$$
s=k_{o} \sigma+e,
$$

where $k_{0}=\left(k_{0,1} k_{0,2}\right)>0$ and $\dot{\sigma}=e$.

Augmenting SMC with conventional integrator will remove the steady-state error at the cost of degradation in transient response. In order to improve the transient response as well as the steady-state response of the system, we will use the technique of augmenting SMC with conditional integrator introduced in [13]. The sliding surface will be the same as defined in expression (19) however the integrator design will be modified as given,

$$
\dot{\sigma}=-k_{0} \sigma+\mu\left(\begin{array}{l}
\operatorname{sat}\left(s_{1} / \mu_{1}\right) \\
\operatorname{sat}\left(s_{2} / \mu_{2}\right)
\end{array}\right)
$$

where $\mu=\left(\mu_{1} \mu_{2}\right)>0$. Using expression (19) and (20) it is obvious that inside the boundary layer when $\{|\mathbf{s}| \leq \mu\}$ then $\dot{\sigma}=-k_{0} \sigma+s=e$, which in turn implies that at equilibrium $e$ will be zero, which means that integral action is applied only conditionally.

\section{Results and Simulations}

In this section, we will present the results obtained through simulations by applying the proposed control law. Though the law designed is based on the linear model but for simulation, we have used the nonlinear model of mini UAV given by expression (1...4). A summary of the customized mini UAV physical parameters is given in [18] is,

Table 2: Physical parameters of the mini UAV.

\begin{tabular}{|l|l|l|}
\hline Parameter & Value & unit \\
\hline Weight & 3.8 & $\mathrm{Kg}$ \\
\hline Span & 2 & $\mathrm{M}$ \\
\hline MAC & 0.2401 & $\mathrm{M}$ \\
\hline Wing surface area & 0.4802 & $\mathrm{M}^{2}$ \\
\hline Moment of inertia $\mathrm{I}_{\mathrm{y} 1}$ & 0.16 & $\mathrm{Kgm}^{2}$ \\
\hline
\end{tabular}

The initial condition for the state variables are $v(0)=16.57 \mathrm{~m} / \mathrm{sec}, \alpha(0)=5.4^{0} \theta(0)=0$ and $q(0)=0$.To clearly demonstrate the statement that conditional integrator based SMC outperform the ideal and integral based SMC we will consider two different cases and will then compare the results obtained by simulation. For the complete simulation process we have used $K_{0}=15, K_{1}=160, K=20, K_{p}=700, q=3 \mathrm{deg} / \mathrm{sed}$ with two different values of $\mu(1$ and 0.1$)$ and 0.1$)$ for a pitch rate $(q)$ of $3 \mathrm{deg} / \mathrm{sec}$.

Case I- Tracking the desired signal when there is no external disturbance i.e the effect coming from external disturbance is zero. 


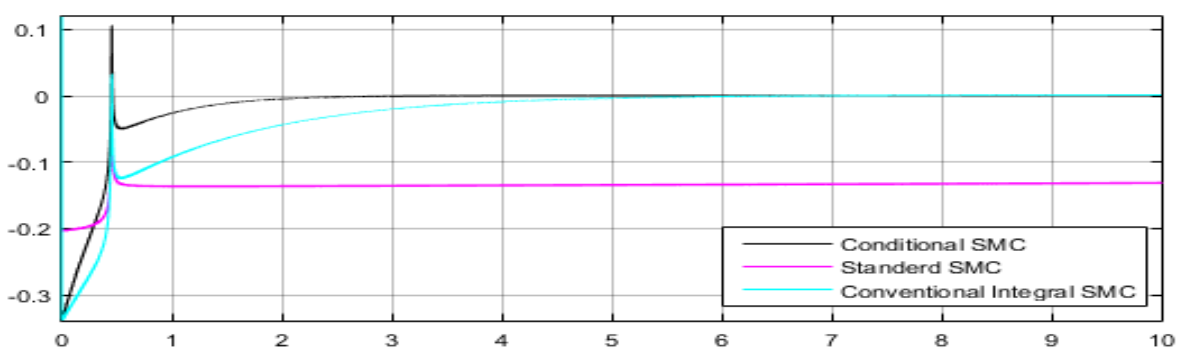

Fig. 1: Tracking error when $\mu=1$.

Figure 1 shows the error in tracking the desired pitch rate, it is clear that the transient, as well as the steady-state error in case of the ideal SMC with saturation function, is more as compared to conventional integral based SMC which has zero steady-state error, however the transient response of the conventional integrator is degraded as compared to that of conditional integral based SMC having zero steady state error.

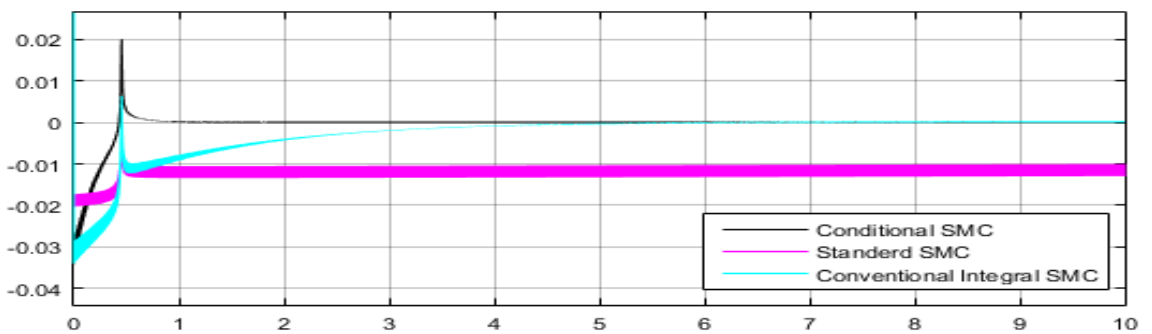

Fig. 2: Tracking error when $\mu=0.1$.

By decreasing the value of $\mu$ these errors can be reduced as shown in figure 2 however this reduction in the value of $\mu$ leads to chattering in case of ideal SMC and conventional integral based SMC as is shown in figure 3. It is clear from the same figure that the response of the conditional integral based SMC is insensitive to change in the value of $\mu$.

Case II- when the system is affected by external disturbances.

In order to show the robustness of the designed controller to the external disturbances, an external disturbance $d(t)$ given by equation (23) is added to the system.

$$
d(t)=\sin (2 t+\pi)+2
$$

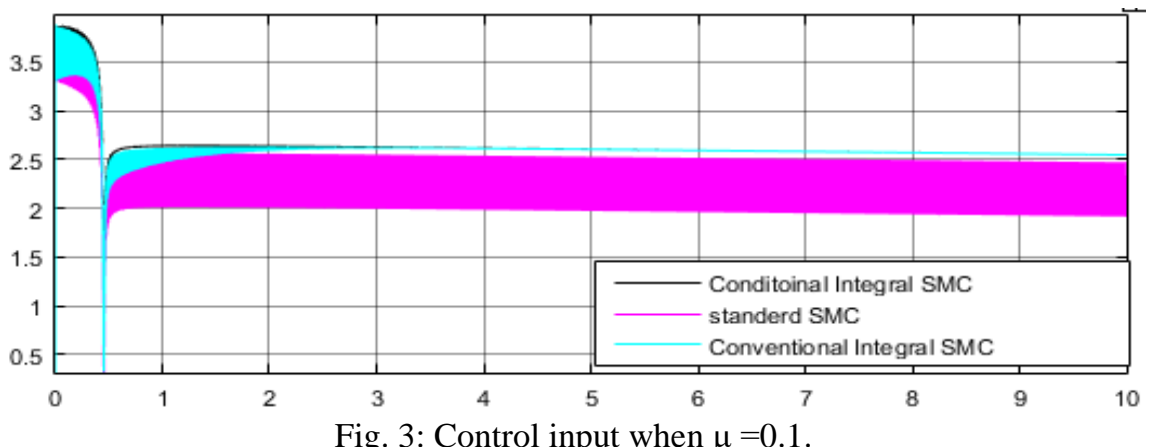

The response curve for tracking the desired pitch rate of the three mentioned controller is shown in figure 4 . From this figure, it is clear that the performance of the conditional integrator based SMC has improved as compared to integral based and standard SMC. 


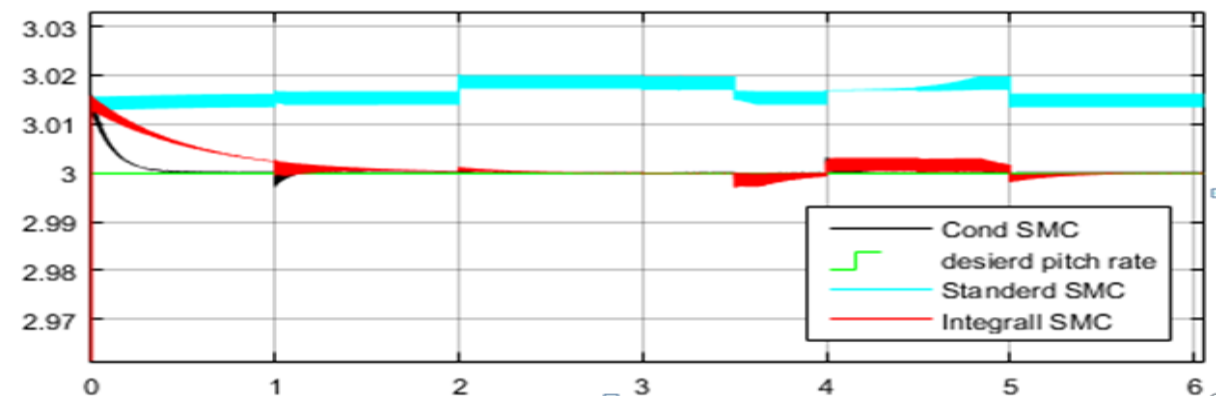

Fig. 4: Tracking of the desired pitch rate in presence of external disturbances with $\mu=0.1$.

Finally, figure 5 shows the performance of the designed control law to track the desired velocity $v_{d}$ in the presence of disturbances and system nonlinearities.

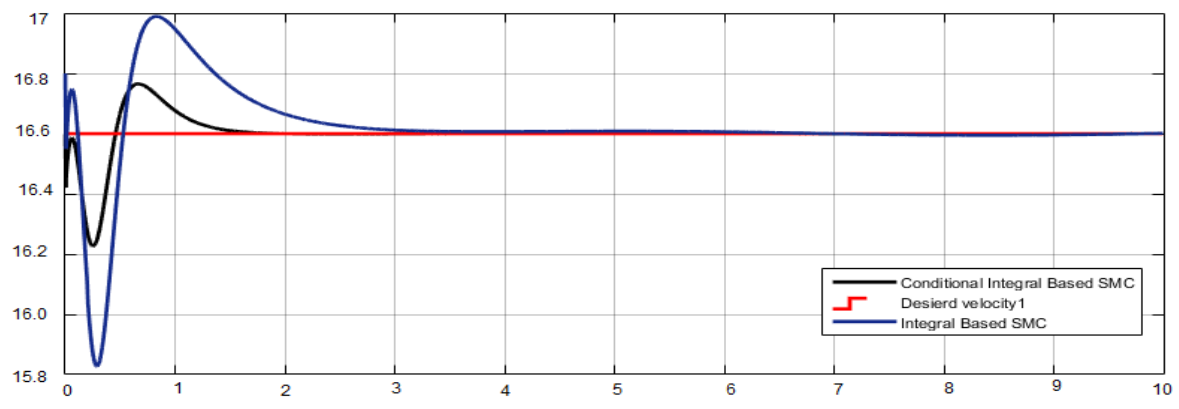

Fig. 5: Velocity tracking using the PI controller.

\section{Conclusion}

In this paper, we have augmented SMC with a conditional integrator for tracking the pitch rate command for a mini UAV. The conditional integrator allows us to eliminate the steady state error without degrading the transient response of the system, by introducing the integral action conditionally. The design of the conditional integrator based SMC exploits the short period mode of the linearized longitudinal dynamics of the mini UAV. The simulation results show that the transient response of the SMC augmented with a conditional integrator is improved as compared to that of an ideal and/or integral based SMC. Also in case of Conditional integrator decreasing the value of $\mu$ reduces the error but without introducing the chattering.

\section{References}

[1] Y. J. Hang, "Sliding Mode Control Design for Aircraft Control Systems," in Aerospace Control Systems, Proceedings. The First IEEE Regional Conference on, Westlake Village, CA, 1993, pp. 309-313, USA.

[2] E. Promtun and S. Seshagiri, "Sliding Mode Control of Pitch-Rate of an F-16 Aircraft," in Proceedings of the 17th World Congress The International Federation of Automatic Control, Seoul Korea, pp. 1099-1104, 2008.

[3] J. Magni, B. Samir and T. Jan, Robust flight control. London, Springer, 1997.

[4] L. Marconi, R. Naldi and A. Isidori, "High-gain output feedback for a miniature UAV," International Journal of Robust and Nonlinear Control, vol. 24, no. 6, pp. 1104-1126, 2013.

[5] R. Bhattacharya, G. J. Balas, M. Kaya and A. Packard, "Nonlinear Receding Horizon Control of an F-16 Aircraft," Journal Of Guidance, Control, And Dynamics, vol. 25, no. 5, pp. 924-931, 2005.

[6] W. Reigelsperger and S. Banda, "Nonlinear simulation of a modified F-16 with full-envelope control laws," Control Engineering Practice, vol. 6, no. 3, pp. 309-320, 1998.

[7] R. Rysdyk and A. Calise, "Robust nonlinear adaptive flight control for consistent handling qualities," IEEE Transactions on Control Systems Technology, vol. 13, no. 6, pp. 896-910, 2005. 
[8] T. Lee and Y. Kim, "Nonlinear Adaptive Flight Control Using Backstepping and Neural Networks Controller," Journal of Guidance, Control, and Dynamics, vol. 24, no. 4, pp. 675-682, 2001.

[9] S. Amini and A. Akbar Akbari, "Adaptive Sliding Mode Controller Design For Attitude Small UAV," International Journal of Robotics and Automation, vol. 4, no. 3, pp. 219-229, 2015.

[10] A. Esna Ashari and H. Khaloozadeh, "Substitute Sliding-Mode Controller Design for Aircraft Control," in Proc. of Industrial Technology, Mumbai, India, pp. 2280-2285, 2006.

[11] H. Xu, M. Mirmirani and P. Ioannou, "Adaptive Sliding Mode Control Design for a Hypersonic Flight Vehicle," Journal of Guidance, Control, and Dynamics, vol. 27, no. 5, pp. 829-838, 2004.

[12] C. Chen, H. Chiang and J. Shen, " A comparison of various VSS techniques on the control of automated guided vehicles," International Journal of Fuzzy Systems, vol. 11, no. 2, pp. 97-106, 2009.

[13] S. Seshagiri and H. Khalil, "Robust output feedback regulation of minimum-phase nonlinear systems using conditional integrators," Automatica, vol. 41, no. 1, pp. 43-54, 2005.

[14] B. Stevens and F. Lewis, Aircraft control and simulation. New York: John Wiley \& Sons, INC, 2004.

[15] B. Pamadi, Performance, stability, dynamics, and control of airplanes, 2nd ed. Reston: American Institute of Aeronautics

[16] J. Slotine and W. Li, Applied nonlinear control. London: Prentice-Hall International (UK) Limited, 1991.

[17] C. Hao, Z. Beiji, Z. Haoyu and P. Lili, "Sliding Mode Controller Design for an Aircraft Pitch Rate Track System," in IEEE International Conference on Network, Sensing and Control, pp. 1004-1007, 2008.

[18] S. Swarnkar, and M. Kothari, "A Simplified Adaptive Backstepping Control of Aircraft Lateral/Directional Dynamics,” IFAC-PapersOnLine, vol. 49, pp. 579-584, 2016. 